\title{
Study on the Migration and Transformation Law of Arsenic in Groundwater, Soil and Other Multi-Media
}

\author{
Jun Hou \\ College of Resources and Environmental Sciences, Xinjiang University, Urumqi, Xinjiang 830046, China
}

Keywords: Arsenic, Groundwater, Soil, Migration transformation

\begin{abstract}
With the development of modern industry and the intensification of human activities, a large number of pollutants are discharged, which makes the environmental quality of the atmosphere, soil, rivers and lakes deteriorate day by day, seriously affecting the sustainable development of industrial and agricultural production and people's life and health. In this paper, field irrigation experiments were carried out in typical areas with high arsenic groundwater distribution, and the effects of irrigation on hydrogeochemical processes and sediment geochemical characteristics of unsaturated zone were simulated. On this basis, the mechanism of arsenic migration and transformation in unsaturated zone under the influence of irrigation activities was discussed. Soil bulk density, soil moisture content, adsorption coefficient, convection velocity, hydrodynamic dispersion velocity, adsorption degradation, emission mode of pollution sources, boundary conditions and other factors affect the migration process of pollutants in soil to varying degrees. Soil has a certain adsorption and purification capacity for arsenic. In the adsorption reaction, the adsorption capacity of arsenic increases with the adsorption time until saturation.
\end{abstract}

\section{Introduction}

Arsenic is a kind of toxic metal element with strong stability, which has a wide range of biological effects. It has been recognized as a carcinogen by human beings by many authoritative organizations such as the Centers for Disease Control (CDO), the International Agency for Cancer Prevention (LARC), the US Environmental Protection Agency (EPA) and the National Toxicology Program [1]. Recent studies have shown that bacteria with arsenic resistance genes can produce gaseous arsenic compounds by biomethylation of a considerable number of water-soluble inorganic arsenic compounds. The toxicity of gaseous arsenic compounds is greater than that of inorganic trivalent arsenic (LC50<3mg/kg)[2]. The discovery of this phenomenon puts forward new problems and research directions for arsenic pollution process and treatment. Experimental study provides a necessary means for qualitative analysis and quantitative study of migration of organic pollutants in porous media. In recent years, many scholars have done a lot of research on migration and transformation of organic compounds in soil, unsaturated zone, groundwater and clay impervious layer, and indoor and outdoor experiments have made great progress.

Arsenic enters human body through respiratory tract, digestive tract and skin contact. Under normal circumstances, there are trace amounts of arsenic in soil, water, air, plants and human body, which will not cause harm to human body. Therefore, it is necessary to systematically study the migration and transformation process of arsenic in groundwater, soil and other multi-media in order to screen out feasible ways to reduce the bioavailability of arsenic and provide scientific theoretical basis for establishing arsenic pollution control index system in polluted areas.

\section{Main Factors Affecting Pollutant Transport}

\subsection{Soil Texture}

Soil texture reflects the composition of soil particles. Soil particles with different textures have different pore size and distribution, which leads to different soil water potential and soil water conductivity, thus affecting the infiltration capacity of soil and the migration speed of pollutants in 
soil. All kinds of organic and inorganic colloids can adsorb arsenic, especially the hydroxides of lead and iron have special affinity for arsenic, and this adsorption capacity is the strongest under acidic conditions. Arsenic reductase exists in Pteris vittata, an arsenic hyperaccumulator, which is mainly distributed in roots. Most of the pentavalent arsenic entering the root cells is reduced to trivalent arsenic by arsenate reductase, and most of the trivalent arsenic is transported to the aerial parts through xylem, and shielded in vacuoles of aerial parts cells in the form of trivalent arsenic, thus achieving the purpose of detoxification. In the process of hazardous waste landfill disposal, groundwater pollution caused by infiltration and migration of harmful components is the most important way for hazardous waste to pollute the environment.

\subsection{Soil Structure}

Various arsenic compounds enter the water through various channels, and their physical actions are largely restricted by the redox conditions in the surrounding environment of the water. Studies have shown that phosphate and arsenate in soil can compete with each other for adsorption sites on soil colloid [4]. Therefore, some studies suggest that phosphate fertilizer can be applied to control and treat arsenic contaminated soil, while some research results show that phosphorus application will increase the bioavailability of arsenic in soil, thus aggravating the harm of arsenic to crops [5]. In addition, because of the variety and complex nature of hazardous wastes, their harm to groundwater is obviously long-term, latent and irreversible. Once groundwater pollution occurs in hazardous waste landfill, it will take decades or even hundreds of years to clean up the groundwater pollution caused by hazardous wastes. If the saturated hydraulic conductivity of the upper and lower soils is quite different, the lower soil forms a water-resisting layer compared with the upper soil, which may form a temporary water level at the interface.

\subsection{Soil Temperature Field}

Soil temperature directly affects soil water form, soil water viscosity and soil surface tension. The change of temperature not only affects the soil hydraulic conductivity, but also affects the state of soil moisture. When the average temperature of soil rises, the infiltration capacity of soil increases. Therefore, trivalent arsenic in surface water is easily oxidized to pentavalent arsenic, which forms arsenic-iron precipitate with ferric hydroxide in water and sinks in sediment. Groundwater pollution is the change of physical, chemical and biological properties of groundwater, which limits its various uses in normal function or hinders its application [6]. Elemental speciation analysis requires modern analytical techniques to analyze and locate the speciation of elements in environmental and biochemical samples. Modern analysis technology requires its advantages of rapidity, convenience, high sensitivity and high resolution. Hydrolysis and complexation process, dissolution and precipitation process, redox process, biochemical process, plant root absorption process, volatilization process, etc. The adsorption of pollutants in soil can be described by partition theory and adsorption theory. Through this process, we can see that the content of arsenic element (simple substance) in water is very low except man-made pollution.

\section{Analysis of the Law of Migration and Transformation in Different Media}

Many researchers believe that the adsorption of pollutants on solid media is a rapid nonequilibrium process, which is complex and almost unpredictable at present, and dispersion restriction seems to play an important role [7]. Every infinitesimal unit of porous media is composed of solid matter and pores, and a new hydrodynamic dispersion model considering the jump change of concentration and concentration gradient at the interface between solid matter and pores is put forward, which leads to the addition of supplementary terms to the hydrodynamic dispersion equation. If the flow rate is too high, it will dilute the gaseous arsenide and make it stay on the optical path for a short time, which will reduce the sensitivity. It will also make the contact time between the gaseous arsenide and the adsorption material too short and can not be completely adsorbed by the adsorption material. The absorption of solute in soil by plant roots depends on the absorption characteristics of roots on the one hand, and on the ability and speed of supplying solute 
to plant roots on the other hand. There are three ways for plant roots to absorb pollutants in soil: It forms insoluble compounds or precipitates with iron and lead hydroxide compounds. However, water-soluble arsenic only accounts for $5 \%-10 \%$ of the total amount. However, some studies also show that the amount of amorphous iron oxide in flooded soil increases greatly, which affects the availability of phosphorus and arsenic in soil. It can be seen that the form of iron in soil can also directly or indirectly affect the form and bioavailability of arsenic in soil [8].

After the construction of irrigation site is completed, the irrigation experiment will be started in the experimental area, and water will be diverted from the pumping well for irrigation. After irrigation, the arsenic concentration in $2.5 \mathrm{~m}$ phreatic water gradually decreased, which indicated that As(III) was still migrating down into deeper aquifer, and the percentage of As(III) in total arsenic decreased, which was due to the reduction condition weakened due to the decrease of phreatic water level.On the whole, the content of total arsenic in $0 \sim 0.5 \mathrm{~m}$ soil pore water is not high, and it is all lower than $10 \mu \mathrm{g} / \mathrm{L}$. However, the arsenic concentration in $2 \mathrm{~m}$ diving (Table 1) rises sharply during irrigation, from $14.33 \mu \mathrm{g} / \mathrm{L}$ before irrigation to more than $100 \mu \mathrm{g} / \mathrm{L}$, and the arsenic form is mainly As (III).

Table 1 Changes of Arsenic Content in 2.5 m Diving during Irrigation

\begin{tabular}{|l|l|l|l|l|l|}
\hline \multirow{2}{*}{ Sampling batch } & Before irrigation & Under irrigation & \multicolumn{2}{|l|}{ After irrigation } \\
\cline { 2 - 6 } & S-0 & S-1 & S-2 & S-3 & S-4 \\
\hline Total $\operatorname{arsenic}(\mu \mathrm{g} / \mathrm{L})$ & 14.33 & 15.62 & 15.39 & 229.61 & 102.71 \\
\hline $\operatorname{As}(\mathrm{III})(\mu \mathrm{g} / \mathrm{L})$ & 13.84 & 12.47 & 14.07 & - & 88.93 \\
\hline $\operatorname{As}(\mathrm{V})(\mu \mathrm{g} / \mathrm{L})$ & 0.37 & 2.81 & 0.82 & - & 19.24 \\
\hline
\end{tabular}

A large number of experimental studies have made the coefficients of attenuation, ion exchange, biological and chemical reactions in the migration equation more comprehensive and reasonable, and considered the mutual transformation relationship between solid and liquid concentration of pollutants, while the adsorption conditions developed from equilibrium isothermal model to nonequilibrium adsorption model. Arsenic generally participates in precipitation reaction and redox reaction in water, and its migration and transformation is relatively simple. Arsenic in soil not only undergoes redox reaction with its environment, but also participates in complexation and chelation reaction with organic matter, and its migration and transformation is more complicated than that in water. There are differences in the migration, transformation and bioavailability of arsenic in soil and groundwater, especially in rhizosphere which has special physical, chemical and biological properties. This process is complex and almost unpredictable at present, and dispersion restriction seems to play an important role. Adsorption kinetics experiment mainly studies the adsorption process of arsenic on specific research medium soil. In the aspect of soil water movement, from the average pore water flow in unsaturated zone to the study of movable and immovable water bodies, and considering the interaction among water, air, pollutants and soil comprehensively. The solution of mathematical model is also developing continuously, from simple analytical solution of unsaturated zone to numerical solution of complex factors. When root interception and mass flow can't provide enough solute for root system, continuous absorption will reduce the effective solute concentration in the soil on the root surface, resulting in the decrease of concentration gradient perpendicular to the root surface and diffusion.

Water $\mathrm{pH}$ is an important factor affecting arsenic enrichment in water. The $\mathrm{pH}$ of soil pore water in irrigation area varies from 7.03 to 8.71, showing weak alkalinity, showing an obvious positive correlation with arsenic (Figure 1). This is because arsenic is easily adsorbed by positively charged minerals and clay minerals in water. When the $\mathrm{pH}$ of soil pore water increases, the adsorption capacity of positively charged minerals and clay minerals to arsenic in soil pore water decreases, and arsenic is desorbed into soil pore water, resulting in arsenic content in soil pore water. 

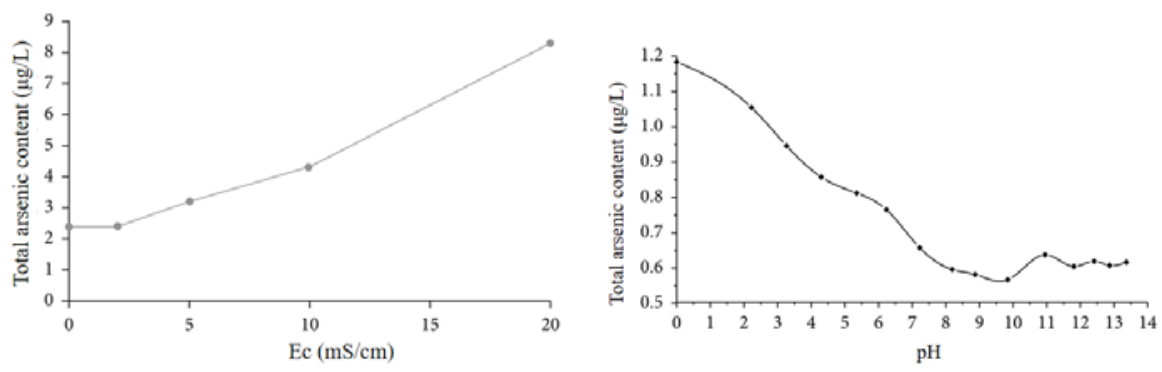

Fig.1 The Relationship between as Concentration and $\mathrm{Ph}(\mathrm{a})$ and $\mathrm{Ec}(\mathrm{B})$ in Soil Pore Water

Infiltration and migration of organic pollutants in the vadose zone is a very complicated process. Insoluble precipitates hinder the penetration of liquid, resulting in lower permeability coefficient than pure water. Due to the existence of groundwater in soil, as well as a large number of organic and inorganic colloids, soil animals and plants, and microorganisms, pollutants in soil are constantly migrated, transformed, adsorbed and decomposed through chemical, physical and biological processes of soil. With the increase of $\mathrm{pH}$ value, the adsorption capacity of arsenic in soil decreases, the water solubility increases, and the pollution increases, whereas the pollution decreases. However, no matter the increase or decrease of $\mathrm{pH}$ and $\mathrm{EH}$ in water, arsenic has little effect on the overall pollution of water.

There are a lot of organic matter in the vadose zone, and it also has a huge specific surface area, which has a strong surface binding effect on pollutants entering the soil environment, including physical, chemical and biological effects. Soil particles in the vadose zone are the main carriers for the migration and transformation of pollutants. In the unsaturated zone near the surface, irrigation makes its reduction environment stronger, some crystalline iron oxides are transformed into amorphous iron oxides, and amorphous iron oxides and dissolved carbonate iron lead to arsenic release. However, $\mathrm{O} 2$ still enters the unsaturated zone near the surface, and $\mathrm{Fe}(\mathrm{II})$ is oxidized to $\mathrm{Fe}(\mathrm{III})$ and then forms crystalline iron oxides, and As(III) is rapidly oxidized to As(V) and fixed by crystalline iron oxides in the form of adsorption/coprecipitation. Indigenous microorganisms in soil can degrade arsenic pollution after acclimation, but the proportion of microbial degradation to arsenic trapped in soil vadose zone is only about $3 \%$.

\section{Conclusion}

In this paper, field irrigation experiments were carried out in typical areas with high arsenic groundwater distribution, and the effects of irrigation on hydrogeochemical processes and sediment geochemical characteristics of unsaturated zone were simulated. On this basis, the mechanism of arsenic migration and transformation in unsaturated zone under the influence of irrigation activities was discussed. Arsenic migrates from non-rhizosphere to rhizosphere with iron oxidation and reduction, and is enriched in rhizosphere. Due to the buffer layer of iron film, arsenic rarely enters the root system of crops, and the arsenic content in aerial parts is the lowest; Arsenic in water is mainly affected by $\mathrm{pH}$ and $\mathrm{EH}$, while arsenic in soil is mainly affected by adsorption of soil colloid and complexation and chelation of heavy metals. Convection is the main factor affecting the migration and transformation of pollutants. Soil bulk density, soil moisture content, soil adsorption distribution coefficient and other factors have certain influence on the migration of pollutants. In the process of pollutant discharge, the concentration will decrease or tend to be flat after reaching a certain value, that is, the concentration has a process of accumulation first, and then a process of release.

\section{References}

[1] Yan Yijun, Xie Xianjun, Zheng Wenjun, et al. Effect of irrigation on migration of arsenic in surface soil of Datong Basin. Geological Science and Technology Information, No. 03, pp. 235-241, 2017. 
[2] Pei Liang, Liao Xiaoyong. Experimental study on the temporal and spatial distribution of arsenic (As) in soil with regenerated drip irrigation. Journal of Water Resources and Water Engineering, Vol. 29, No. 02, pp. 239-242, 2018.

[3] Yan Yijun, Xie Xianjun, Zheng Wenjun, et al. Influence of Irrigation Practices on Arsenic Mobilization in Near-Surface Soil of Datong Basin, Northern China. Geological Science and Technology Information, Vol. 036, No. 003, pp. 235-241, 2017.

[4] Yang Lanpeng, Li Na, Zhang Jun. The effect of $\mathrm{pH}$ on the migration and transformation of “trinitrogen” in shallow groundwater. Chinese Agricultural Science Bulletin, Vol. 033, No. 030, pp. 56-60, 2017.

[5] Liu Yaci, Li Yasong, Zhang Zhaoji, et al. Distribution rule of arsenic compounds and groundwater arsenic compounds around chicken farms in Lubei Plain. South-to-North Water Transfer and Water Conservancy Science and Technology, Vol. 15, No. 003, pp. 86-93, 2017.

[6] Tai Tuoya, Zheng Yuejun, Wang Jinsheng. Application of HYDRUS-1D model to simulate and analyze the migration characteristics of PFCs in soil. Journal of Agricultural Environmental Sciences, Vol. 37, No. 10, pp. 91-98, 2018.

[7] Gu Tao, Zhao Xinwen, Wang Jietao, et al. Distribution characteristics of cadmium, mercury and arsenic elements in a paddy field water-soil-plant system. Safety and Environmental Engineering, Vol. 24, No. 006, pp. 70- 75, 2017.

[8] Yan Yijun, Xie Xianjun, Xiao Ziyi, et al. Effect of irrigation on migration and transformation of arsenic in unsaturated zone. Geological Science and Technology Information, Vol. 37, No. 05, pp. 212-220, 2018. 